\title{
Retrieving the optical potential from lattice simulations
}

\author{
Maxim Mai* ${ }^{*}$ \\ Institute for Nuclear Studies and Astronomy, Physics, and Statistics Institute of Science, \\ Department of Physics, The George Washington University, $72521^{\text {st }}$ St. NW, Washington, DC \\ 20052, USA \\ E-mail: maximmai@gwu.edu
}

\section{Michael Döring}

Institute for Nuclear Studies and Astronomy, Physics, and Statistics Institute of Science, Department of Physics, The George Washington University, $72521^{\text {st }}$ St. NW, Washington, DC 20052, USA;

Thomas Jefferson National Accelerator Facility, 12000 Jefferson Ave, Newport News, VA 23606, USA

\section{Dimitri Agadjanov}

Helmholtz-Institut für Strahlen- und Kernphysik (Theorie) and Bethe Center for Theoretical Physics, Universität Bonn, D-53115 Bonn, Germany

\section{Ulf-G. Meißner}

Helmholtz-Institut für Strahlen- und Kernphysik (Theorie) and Bethe Center for Theoretical Physics, Universität Bonn, D-53115 Bonn, Germany;

Institute for Advanced Simulation (IAS-4), Institut für Kernphysik (IKP-3) and Jülich Center for Hadron Physics, Forschungszentrum Jülich,

D-52425 Jülich, Germany

\section{Akaki Rusetsky}

Helmholtz-Institut für Strahlen- und Kernphysik (Theorie) and Bethe Center for Theoretical Physics, Universität Bonn, D-53115 Bonn, Germany

We propose a method for the direct extraction of the complex hadron-hadron optical potential on the lattice, which does not require the use of the multi-channel Lüscher formalism and can be applied to systems with three and more particles. The method is tested on a set of realistic synthetic data for a two channel problem.

34th annual International Symposium on Lattice Field Theory

24-30 July 2016

University of Southampton, $U K$

\footnotetext{
${ }^{*}$ Speaker.

${ }^{\dagger}$ The speaker wishes to thank the organizers for invitation and partial financial support. He also wishes to thank the German Research Foundation (DFG) for financial support. M.D. acknowledges financial support from the National Science Foundation (NSF), CAREER grant No. PHY-1452055 and the Department of Energy (DOE), office of Nuclear Physics under contract DE-AC05-06OR23177.
} 


\section{Introduction}

The Lüscher approach [1] has become a standard tool to study hadron-hadron scattering processes on the lattice. The use of this approach in case of elastic scattering is conceptually straightforward: besides technical complications, caused by partial-wave mixing, each measured energy level at a given volume and in a given frame of motion uniquely determines the value of the elastic phase shift at the same energy.

In the presence of multiple channels, the extraction of the scattering phase becomes more involved. In case when only two-particle coupled channels appear, one can make use of the coupledchannel Lüscher formalism $[2,3,4,5,6,7,8,10]$ and fit a simple energy dependent parameterization for the multi-channel $K$-matrix elements to the measured energy spectrum in the finite volume [9]. A more sophisticated parameterization of the $K$-matrix elements, which is applicable in a wider range of the energies, can be obtained using unitarized chiral perturbation theory (ChPT) $[10,11,12]$. In the one-channel case such an approach has been successfully applied, e.g., in Refs. $[13,14]$ to analyze $P$-wave $\pi \pi$ scattering and to study the properties of the $\rho$-meson. However, in order to include the coupled channels $\pi \pi-K \bar{K}$ above $K \bar{K}$ threshold, one has to determine three $K$-matrix elements (unknowns) from a single measurement of a finite-volume energy level. Hence, using some kind of (phenomenology-inspired) parameterizations of the multi-channel $K$ matrix elements becomes inevitable in practical applications.

In case when some of the inelastic channels contain three or more particles, the situation is far more complicated. Despite the recent progress in the formulation of the theoretical framework [15, $16,17,18]$, it is still too cumbersome to be directly used in the analysis of the data. Moreover, the problem of the choice of the parameterization for three-particle scattering might become more difficult (and lead to even larger theoretical uncertainties) than in two-particle scattering.

From the above discussion it is clear that a straightforward extension of the Lüscher approach through the inclusion of more channels has its limits that are reached rather quickly. On the other hand, many interesting systems, which are already studied on the lattice, may decay into multiple channels. In our opinion, the present situation warrants a rethinking of the paradigm. One may for example explore the possibility to analyze the lattice data without explicitly resolving the scattering into each coupled channel separately. Such a detailed information is usually not needed in practice. Instead, in the continuum scattering problem, the effect of inelastic channels could be included in the so-called optical potential $[19,20]$, whose imaginary part is non-zero due to the presence of the open inelastic channels. In many cases, it would be sufficient to learn how one extracts the real and imaginary parts of the optical potential from the lattice data, without resorting to the multi-channel Lüscher approach. In the present work, we propose such a method, which relies on the knowledge of a sufficiently large number of eigenvalues measured in lattice simulations. Furthermore, we suggest a method that allows one to obtain this set of eigenvalues by varying a continuous parameter - the twisting angle that defines the boundary conditions set on the quark fields in the simulations [21, 22, 23, 24, 25]. The latter has its own limitations, but there exist certain systems, where it could in principle be applied. One of such examples is the scattering in the coupled-channel $\pi \eta-K \bar{K}$ system in the vicinity of the $K \bar{K}$ threshold and the $a_{0}(980)$ resonance, see also the recent lattice QCD simulation and analysis of this system in Refs. [14, 26]. For further examples, we refer the reader to the original publication [27]. 


\section{Optical potential in the Lüscher approach}

The method proposed in this work, relies on the Feshbach projection operator technique [19]. In continuum scattering theory, it allows to include the inelastic channels effectively in the socalled optical potential. While the general derivation of this method is presented in the original publication [27], we wish to demonstrate this method here on the example of two-particle scattering amplitude parametrized by the so-called $K$ matrix. Further, we restrict ourselves for simplicity to a specific system of two two-particle states, namely $K \bar{K}$ and $\pi \eta$ in S-wave. In this setting the $T$-matrix, related to the unitary $S$-matrix via $S=\mathbb{1}-i T$, is given as a function of center-of-mass energy $(E)$ by

$$
T(E)=\frac{1}{K^{-1}(E)-i \operatorname{diag}\left\{p_{K \bar{K}}, p_{\pi \eta}\right\}},
$$

where $T$ and $K$ are two-by-two matrices, and $p_{\ldots}$ denotes the modulus of the center-of-mass system three momentum in the corresponding two-particle system. For the connection of this equation to the usual Lippmann-Schwinger equation we refer to Ref. [27].

We divide now the multi-channel scattering problem into a primary channel (in our example, the $K \bar{K}$ channel) and a secondary channel, subsuming all other channels, potentially including multi-particle states (in our example, the $\pi \eta$ channel). The $T$-matrix element of this channel is then simply given by

$$
T_{K \bar{K} \rightarrow K \bar{K}}(E)=\frac{1}{W^{-1}(E)-i p_{K \bar{K}}} \quad \text { for } \quad W^{-1}(E)=M_{K \bar{K} \rightarrow K \bar{K}}-\frac{M_{K \bar{K} \rightarrow \pi \eta}^{2}}{M_{\pi \eta \rightarrow \pi \eta}-i p_{\pi \eta}} .
$$

Here $M$ denotes the inverse of the $K$ matrix, which is smooth even if the latter might have poles for real energies. The optical potential $W(E)$ contains all in-elasticities from the secondary channel $(\pi \eta)$ and is therefore a complex valued function above the $\pi \eta$ threshold.

In a finite volume, the momenta are discretized and so is the spectrum of the $T$-matrix. The real-valued poles of the latter determine the energy eigenvalues of the system in finite volume measured in a lattice simulation. Their positions are determined from the following secular equation

$$
W_{L}^{-1}(E):=\frac{2}{\sqrt{\pi} L} Z_{00}\left(1 ; q_{K \bar{K}}^{2}(E)\right)=M_{K \bar{K} \rightarrow K \bar{K}}(E)-\frac{M_{K \bar{K} \rightarrow \pi \eta}^{2}(E)}{M_{\pi \eta \rightarrow \pi \eta}(E)-\frac{2}{\sqrt{\pi} L} Z_{00}\left(1 ; q_{\pi \eta}^{2}(E)\right)},
$$

which is equivalent to Eq. (2.2) when imposing periodic boundary conditions in a cubic box of size $L$, thus, replacing $i p_{k} \rightarrow \frac{2}{\sqrt{\pi} L} Z_{00}\left(1 ; q_{k}^{2}\right)$ with $q_{k}=\frac{p_{k} L}{2 \pi}$ for a corresponding channel $k$. Here, $Z_{00}$ denotes the Lüscher zeta-function. The first equality in Eq. (2.3) determines the finite-volume $K \bar{K}$ optical potential as a function of measured eigenenergies of the system, potentially containing not only the $\pi \eta$ channels but also multi-meson states and other allowed two-body channels. The second equality holds only in our specific two-channel example and shows how the optical potential is related to the $\pi \eta$ channel.

For every eigenvalue $E$, measured on the lattice the left-hand-side of the Eq. (2.3) coincides with the cotangent of the so-called pseudophase which defines the inverse of the finite volume counterpart of the optical potential $W_{L}^{-1}(E)$ at this energy. Obviously the latter is a real-valued function and the main question is now, how to relate it to its infinite-volume counterpart. The latter, 
complex-valued potential $W^{-1}$, is directly related to the $T$-matrix element in infinite volume via Eq. (2.2). It is important to note that, if $L$ tends to infinity, the optical potential does not have a well-defined limit at a given energy. In this limit, the energy levels in the secondary channel(s) condense towards the threshold such that the quantity $W_{L}^{-1}(E)$ oscillates from $-\infty$ to $+\infty$ at any fixed $E$. As a matter of fact, this issue has already been addressed in the literature in the past considering adiabatic switching of the interaction, see Ref. [28]. This is equivalent to attaching an infinitesimal imaginary part $E \rightarrow E+i \varepsilon$ to the energy. Further, as argued in Ref. [28], the limits $L \rightarrow \infty$ and $\varepsilon \rightarrow 0$ are not interchangeable. A correct infinite-volume limit is obtained, when $L \rightarrow \infty$ is performed first, see the discussion in the original publication [27].

Practically, for a given set of energy eigenvalues $\left\{E_{i} \mid i \in \mathbb{N}\right\}$ the above prescription can be performed in three steps: 1) Analytic continuation into complex energy-plane can be performed fitting the set of the pairs $\left\{\left(E_{i}, W_{L}^{-1}\left(E_{i}\right)\right) \mid i \in \mathbb{N}\right\}$ by a sum of simple poles plus a regular background, i.e. $\hat{W}_{L}^{-1}(E)=\sum_{i} \frac{Z_{i}}{E-Y_{i}}+D_{0}+D_{1} E+D_{2} E^{2}+D_{3} E^{3}$, replacing then $E \rightarrow E+i \varepsilon$. The order of the polynomial part in $W_{L}^{-1}$ is not fixed a priori, whereas the general form is valid even when multi-particle intermediate states are involved, as shown in Ref. [27] using time-ordered perturbation theory arguments. 2) The poles of $W_{L}^{-1}(E)$ on the real energy axis lead to oscillations for complex energies. The frequency of these is related to the number of poles in a given energy region. Thus, it rises with increasing lattice volume. However, the amplitude of those tends to zero when $L \rightarrow \infty$. Therefore, the latter limit can simply be obtained "smoothing" over these oscillations at a fixed value of $\varepsilon .3$ ) Finally, the extrapolation of the smoothed $W_{L}^{-1}(E+i \varepsilon)$ to real energies $(\varepsilon \rightarrow 0)$ yields the infinite volume limit of the optical potential.

\section{Application of the method}

The method described in the last section allows to retrieve the $T$ matrix element from the a set of energy eigenvalues in a given channel. The latter is possible irrespectively of the ordering of the primary and secondary channels and is valid even if the secondary channels contain multi-particle states. Technically, one difficulty can arise from the fact that a large number of energy eigenvalues has to be measured to fix the large number of free parameters in $\hat{W}_{L}^{-1}(E)$. However, the change of lattice parameters, such as the reference frame or the box size, alters the optical potential $W_{L}^{-1}(E)$ in finite volume as well. Thus, all energy eigenvalues have to be measured at fixed lattice parameters ${ }^{1}$.

Another alternative is to use a knob, which allows for an energy scan leaving other parameters of the pseudophase intact. In certain systems such a knob is provided by the use of twisted boundary conditions. One of such a system is the coupled-channel $\pi \eta-K \bar{K}$ scattering. As it was discussed in Refs. [3, 25], in this system it is possible to apply (partially) twisted boundary conditions for example, by twisting the light $u, d$ quarks by the same angle and leaving the $s$-quark with periodic boundary conditions. Thus, the $K \bar{K}$ threshold moves and the left-hand-side of the second equality in the secular equation (2.3) changes, while the right one does not.

In the following, we will reconstruct the optical potential from a synthetic lattice data set generated by the chiral unitary approach of Ref. [29]. To extract the potential in the fairly wide energy range from $E=2 M_{K}$ to $E=1.7 \mathrm{GeV}$ we use twisted boundary conditions as described

\footnotetext{
${ }^{1}$ The work on simultaneous analysis of eigenvalues, measured for different lattice parameters is ongoing.
} 


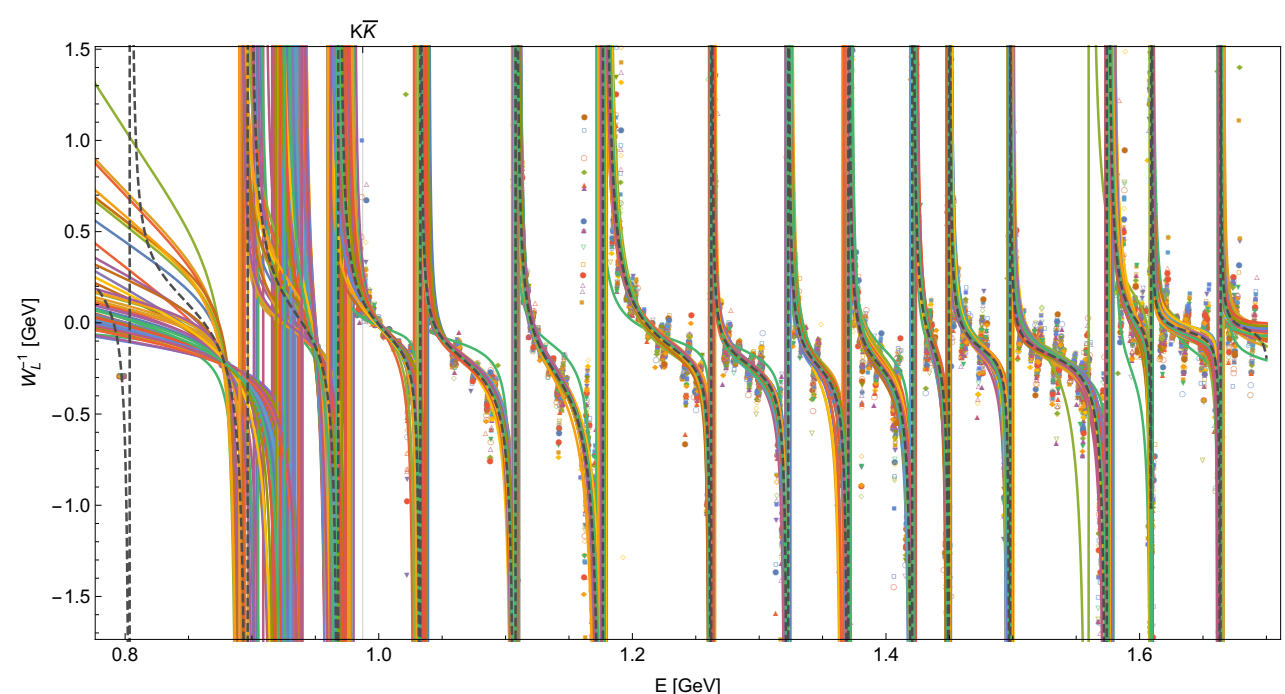

Figure 1: Subset (75 sets) of the re-sampled synthetic lattice data, where each type of marker symbols shows the set of 189 energy eigenvalues, randomly distributed with $\Delta E=1 \mathrm{MeV}$ around the central energy eigenvalues, extracted from Eq. (2.3) imposing twisted boundary condition. Different curves represent fits of $\hat{W}_{L}^{-1}$ to different sets of re-sampled synthetic lattice data. The gray dashed line shows the actual amplitude $W_{L}^{-1}(E)$ to guide the eye.

above. We take six different twisting angles into account, whereas the box size is fixed to be $L=5 M_{\pi}^{-1}$. Solving Eq. (2.3) with $Z_{00}\left(1 ; q_{K \bar{K}}^{2}\right)$ replaced by $Z_{00}^{\theta}\left(1 ; q_{K \bar{K}}^{2}\right)$ for each of the twisting angles we are able to extract 186 energy eigenvalues above and 3 below the $K \bar{K}$ threshold. Note that in this first study, we are interested to test the feasibility of the approach. In an actual lattice simulation one might restrict the energy region, where less energy eigenvalues will be required to reconstruct the optical potential reliably.

Further, in any realistic lattice simulation, the eigenvalues will be known only up to a finite precision, given by $\Delta E$. We study how this error propagates in the proposed approach, starting from a sufficiently large number $(\sim 1000)$ of re-sampled lattice data sets. For each of these sets, normally distributed around the (189) synthetic eigenvalues with a standard deviation of $\Delta E$, we fit the free parameters of $\hat{W}_{L}^{-1}$ minimizing $\chi_{\text {d.o.f. }}^{2}$ as specified in Ref. [27]. We found hereby, that in the present setting (primary channel being heavier than the secondary one) the inclusion of the first pole below the primary threshold stabilizes the fits drastically. Further, we have tested that increase of the polynomial rank in the definition of $\hat{W}_{L}^{-1}$ does not lead to a substantial improvement of the fits. A subset of the results of these fits is depicted in Fig. 1.

In the second step of the procedure, see the end of the previous section, we extrapolate each of the obtained functions $\hat{W}_{L}^{-1}(E)$ to complex energies, then smoothing over the oscillations. For the latter we utilize Gaussian smearing as well as the parametric method as discussed in detail in the original publication [27]. Finally, in the third step, we extrapolate each of the obtained infinite volume results back to the real energies.

The final result of this procedure using parametric smearing and $\Delta E=1 \mathrm{MeV}$ is depicted in Fig. 2. Results using higher $\Delta E$ can be found in the original publication, showing that for not too large $\Delta E$ the error on final result grows linearly with $\Delta E$, but much faster for even higher uncertainties $(\Delta E \gtrsim 10 \mathrm{MeV})$. Further, we have found that both smearing methods lead to essentially the same results. 

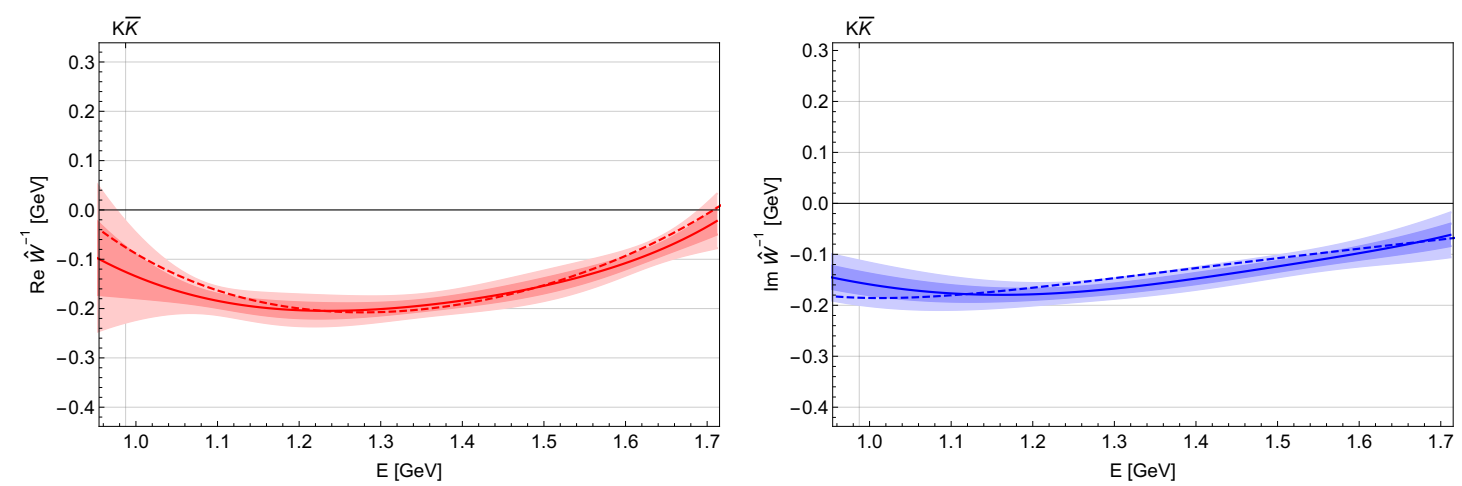

Figure 2: Results of the smearing and extrapolation to real energies using parametric method. The full lines show the average of the re-sampling of all sets, whereas the darker (lighter) bands show the corresponding 1 (2) $\sigma$ error bands. The exact infinite volume solution is shown by the dashed lines for comparison.

\section{Summary}

In the present paper, we formulate a framework for the extraction of the complex-valued optical potential, which describes hadron-hadron scattering in the presence of the inelastic channels, from the energy spectrum of lattice QCD. An optical potential is obtained by using the causal prescription $E \rightarrow E+i \varepsilon$ for the continuation into the complex energy plane. It converges to the "true" optical potential in the limit $L \rightarrow \infty, \varepsilon \rightarrow 0$. A demonstration of the effectiveness of the method has been carried out by utilizing synthetic data.

We would like to emphasize that the use of twisted boundary conditions is only a tool, which is used to perform a continuous energy scan of a certain interval. Whatever method is used to measure the dependence of the pseudophase on energy (all other parameters fixed), our approach, based on the analytic continuation into the complex plane, could be immediately applied.

The approach could be most useful to analyze systems, in which the inelastic channels contain three or more particles. Whereas direct methods based on the use of multi-particle scattering equations in a finite volume will be necessarily cumbersome and hard to use, nothing changes, if our approach is applied. The reason for this is that, in case of an intermediate state with any number of particles, the single poles are the only singularities in any Green's function in a finite volume.

\section{References}

[1] M. Lüscher, Nucl. Phys. B 354 (1991) 531.

[2] M. Lage, U.-G. Meißner and A. Rusetsky, Phys. Lett. B 681, 439 (2009) [arXiv:0905.0069 [hep-lat]].

[3] V. Bernard, M. Lage, U.-G. Meißner and A. Rusetsky, JHEP 1101 (2011) 019 [arXiv:1010.6018 [hep-lat]].

[4] C. Liu, X. Feng and S. He, JHEP 0507 (2005) 011 [arXiv:0504019[hep-lat]]; Int. J. Mod. Phys. A 21 (2006) 847 [[arXiv:0508022[hep-lat]].

[5] M. T. Hansen and S. R. Sharpe, Phys. Rev. D 86 (2012) 016007 [arXiv:1204.0826 [hep-lat]].

[6] R. A. Briceno and Z. Davoudi, Phys. Rev. D 88 (2013) 9, 094507 [arXiv:1204.1110 [hep-lat]]. 
[7] N. Li and C. Liu, Phys. Rev. D 87 (2013) 014502 [arXiv:1209.2201 [hep-lat]].

[8] P. Guo, J. Dudek, R. Edwards and A. P. Szczepaniak, Phys. Rev. D 88 (2013) 014501 [arXiv:1211.0929 [hep-lat]].

[9] J. J. Dudek et al. [Hadron Spectrum Collaboration], Phys. Rev. Lett. 113 (2014) 18, 182001 [arXiv:1406.4158 [hep-ph]]; D. J. Wilson, J. J. Dudek, R. G. Edwards and C. E. Thomas, Phys. Rev. D 91 (2015) 5, 054008 [arXiv:1411.2004 [hep-ph]];

J. J. Dudek, R. G. Edwards and D. J. Wilson, [arXiv:1602.05122 [hep-ph]].

[10] M. Döring, U.-G. Meißner, E. Oset and A. Rusetsky, Eur. Phys. J. A 47 (2011) 139 [arXiv:1107.3988 [hep-lat]].

[11] M. Döring, U.-G. Meißner, E. Oset and A. Rusetsky, Eur. Phys. J. A 48 (2012) 114 [arXiv:1205.4838 [hep-lat]].

[12] A. M. Torres, L. R. Dai, C. Koren, D. Jido and E. Oset, Phys. Rev. D 85 (2012) 014027 [arXiv:1109.0396 [hep-lat]]; M. Döring and U.-G. Meißner, JHEP 1201 (2012) 009 [arXiv:1111.0616 [hep-lat]]. M. Döring, M. Mai and U.-G. Meißner, Phys. Lett. B 722 (2013) 185 [arXiv:1302.4065 [hep-lat]].

[13] D. R. Bolton, R. A. Briceno and D. J. Wilson, [arXiv:1507.07928 [hep-ph]].

[14] D. Guo, A. Alexandru, R. Molina and M. DÃúring, Phys. Rev. D 94 (2016) no.3, 034501 [arXiv:1605.03993 [hep-lat]].

[15] K. Polejaeva and A. Rusetsky, Eur. Phys. J. A 48 (2012) 67 [arXiv:1203.1241 [hep-lat]].

[16] R. A. Briceno and Z. Davoudi, Phys. Rev. D 87 (2013) 094507 [arXiv:1212.3398 [hep-lat]].

[17] M. T. Hansen and S. R. Sharpe, Phys. Rev. D 90 (2014) 11, 116003 [arXiv:1408.5933 [hep-lat]]; M. T. Hansen and S. R. Sharpe, Phys. Rev. D 92 (2015) 11, 114509 [arXiv:1504.04248 [hep-lat]].

[18] U. G. Meißner, G. Rios and A. Rusetsky, Phys. Rev. Lett. 114 (2015) no.9, 091602 Erratum: [Phys. Rev. Lett. 117 (2016) no.6, 069902] [arXiv:1412.4969 [hep-lat]].

[19] H. Feshbach, Annals Phys. 5 (1958) 357; Annals Phys. 19 (1962) 287 [Annals Phys. 281 (2000) 519].

[20] A. K. Kerman, H. McManus and R. M. Thaler, Annals Phys. 8 (1959) 551 [Annals Phys. 281 (2000) 853].

[21] P. F. Bedaque, Phys. Lett. B 593 (2004) 82 [arXiv:0402051[nucl-th]].

[22] C. T. Sachrajda and G. Villadoro, Phys. Lett. B 609 (2005) 73 [arXiv:0411033[hep-lat]].

[23] G. M. de Divitiis, R. Petronzio and N. Tantalo, Phys. Lett. B 595 (2004) 408 [arXiv:0405002[hep-lat]]; G. M. de Divitiis and N. Tantalo, arXiv:0409154[hep-lat].

[24] P. F. Bedaque and J.-W. Chen, Phys. Lett. B 616 (2005) 208 [arxiv:0412023[hep-lat]].

[25] D. Agadjanov, U.-G. Meißner and A. Rusetsky, JHEP 1401 (2014) 103 [arXiv:1310.7183 [hep-lat]].

[26] J. J. Dudek et al. [Hadron Spectrum Collaboration], Phys. Rev. D 93 (2016) no.9, 094506 [arXiv:1602.05122 [hep-ph]].

[27] D. Agadjanov, M. Doring, M. Mai, U.-G. Meißner and A. Rusetsky, JHEP 1606 (2016) 043 [arXiv:1603.07205 [hep-lat]].

[28] B. S. DeWitt, Phys. Rev. 103 (1956) 1565.

[29] J. A. Oller and E. Oset, Nucl. Phys. A 620 (1997) 438 [Nucl. Phys. A 652 (1999) 407] [arXiv: 9702314[hep-ph]]. 